Eldabi, Balaban, Brailsford, Mustafee, Nance, Onggo, Sargent

\title{
HYBRID SIMULATION: HISTORICAL LESSONS, PRESENT CHALLENGES AND FUTURES
}

\author{
Tillal Eldabi \\ Brunel University \\ Brunel Business London \\ Uxbridge \\ Middx, UB8 3PH, UK
}

Mariusz Balaban

MYMIC LLC

1040 University Blvd

Suite 100

Portsmouth, VA 23703, USA

Navonil Mustafee

University of Exeter

Exeter Business School

University of Exeter

Exeter, EX4 4ST, UK

Bhakti Stephan Onggo

Lancaster University

Lancaster University Management School

Lancaster

Lancashire, LA1 4YX, UK
Sally Brailsford

Southampton Business School

University of Southampton

Highfield

Southampton, SO17 1BJ, UK

\author{
Richard Nance \\ Virginia Tech \\ Department of Computer Science \\ 6995 Malinda Road \\ Salem, VA 24153, USA
}

Robert Sargent

Syracuse University

Dept. Electrical Engineering and CS

621 Skytop Road

Syracuse, NY 13244, USA

\begin{abstract}
Hybrid simulation comes in many shapes and forms. It has been argued by many researchers that hybrid simulation provides a better insight of the system in hand as it allows modelers to assess its inherent problems from different dimensions. As a result Hybrid Simulation is becoming an important field within the Modeling and Simulation arena. Yet we find that there no clear and cohesive definition for it. Therefore, this panel paper aims to explore the concept of Hybrid Simulation and its progression through the years. In doing so, we hope to lay out the underpinnings of a structured Hybrid Simulation approach by providing historical narratives of the origins of hybrid models; the current challenges expressed by scholars; and future studies to ensure more focused development of a comprehensive methodology for Hybrid Simulation.
\end{abstract}




\section{INTRODUCTION}

Hybrid simulation comes in many shapes and forms. For example, there are hybrid models that are based on two or more simulation models such as linking discrete event simulation (DES) with either system dynamics (SD) or agent based (ABS) models. Another example of a hybrid study is the combined application of simulation with analytical techniques from the wider Operations Research domain, as also from disciplines such as Systems Engineering and Applied Computing (Powell and Mustafee, 2014) - this is referred to as hybrid systems modelling approach. It has been argued by many researchers that hybrid simulation provides a better insight of the system in hand as it allows modelers to assess its inherent problems from different dimensions (Zulkepli and Eldabi 2015); similarly, research that extends either a traditional or a hybrid simulation study to include theories, methods and practices specific to other disciplines will benefit from having recourse to knowledge constructs that have not traditionally been applied in our field (Mustafee et al., 2015). Therefore, and with the ever rising complexity of the modern world, Hybrid Simulation and hybrid systems modelling approach are both becoming an important field within the Modeling and Simulation arena. Despite the continuous effort in Hybrid Simulation attempts, there are still three main challenges: first, there no clear and cohesive definition for it - e.g. hybrid, combined, and mixed (Lynch et al 2014), hybrid simulation versus hybrid M\&S study (Mustafee et al., 2015). Second, there is a lack of a specific philosophical view point that defines Hybrid Simulation as an independent approach (Shanthikumar and Sargent 1983). This is possibly due to the very nature of hybridization where models are based on mixing several paradigms, making it difficult to be housed within one. The third challenge, which is possibly resulting from the above two, is that most attempts are ad hoc and pragmatic with no clear methodology (Zulkepli and Eldabi 2015). Therefore, this panel paper aims to explore the concept of Hybrid Simulation and its progression through the years. In doing so, we hope to lay out the underpinnings of a structured Hybrid Simulation approach by providing historical narratives of the origins of hybrid models (technological and analytical), the current challenges expressed by scholars and future studies to ensure more focused development of a comprehensive methodology for hybrid models.

\section{HYBRID FRAMEWORKS (ELDABI)}

Linking two or more models at the conceptual level is still a challenging issue despite significant developments in hybridization at the implementation phase. Most of the conceptual hybrid attempts so far have been rather pragmatic with no clear guidelines beyond the specific cases for which they were developed. Having said that, there are some attempts to develop hybrid frameworks for developing models at both the conceptual phase and the implementation phase. These frameworks, however, are yet to pass the threshold of being comprehensive enough to capture all the elements of building a complete hybrid model. This section aims at reviewing existing attempts to build such a framework - mostly proposed over the last decade - in order to envision the building blocks of a more comprehensive framework that takes into account non-technical users (i.e. those with no software development experience) and enable them to develop hybrid models from inception to completion. Whilst the list of the following frameworks is not necessarily exhaustive, it represents more or less the main attempts to develop a hybrid simulation model. For the purpose of this panel, these would suffice to discuss the current scene of hybrid frameworks.

One of the earliest attempts to develop a hybrid framework within the current surge of hybrid simulation was the one proposed by Helal et al. (2007), which introduced a methodology integrating and synchronizing DES and SD applications in an manufacturing systems. Helal's framework is based on a modular concept where the modeled system is decomposed into several smaller modules for modelling purposes. These modules (whether SD and/or DES based) are formalized and synchronized using the SDDES controller. On the other hand, Chahal and Eldabi (2008) proposed a similar framework, which was mainly focused on deciding whether to opt for developing a hybrid model or not. This framework was one of the first one that attempted to identify the "hooks" that link between the hybridized modes at the conceptual phase. Chahal and Eldabi (2008)'s framework focused on linking SD and DES yet it did not go beyond the initial phases 
of identifying the need for hybridization. This framework was extended by Zulkepli and Eldabi (2015) by establishing a three-phased framework that takes into account the initial selection process, followed by a second phase into identifying hybridized models as "modules". The third phase encapsulate a set of steps to help the user to identify the "hooking" variables. Lynch et al (2014) proposed a similar framework that has similar intentions. It suggests a set of "selection" criteria for hybridize with similar objectives to those of Chahal and Eldabi (2008) and Zulkepli and Eldabi (2015), yet adding extra details. Even though no clear guidance is given for the selection criteria, this framework goes into a great length in identifying such criteria coupled with a detailed description of the factors that control the resolution of the model(s). The framework proposed by Lynch et al (2014) provides a set of guidelines for selecting appropriate paradigms given different facets and resolutions of the model. The framework also involves the verification and calibration of the models. Lynch et al (2014) focused their framework on SD, DES, and ABM. Fakhimi et al. (2015) have proposed the Hybrid Simulation Framework for modelling of the Triple Bottom Line (HSFTBL), a framework that focuses on sustainability analysis. Another framework, which was proposed by Morgan et al (2011), lays the seed for an even more comprehensive toolkit for hybridization. The framework is divided into four main components: Problem and System Exploration for assessing the system and the problem for methodological selection and design. This phase use the classical problem structuring approaches. Second, Selection and Characterization for assessing the benefits a combined approach might provide over a single approach. Third, Designing and Combining for assessing how to address the modeling questions. Fourth, Model Development for developing the model(s) according to agreed design. This framework looked at SD and DES.

Although the above frameworks approach hybridization in many different ways, they do agree on the importance of deciding on the need for hybridization. This is a very important step to address as most of the previous attempts do not provide enough guidance. Whilst these frameworks go into a great deal of depth in the selection process and the levels of interactions between the hybridized models, they fall short of providing a single comprehensive framework that encapsulates all of the modelling steps in a simplified fashion. So, in order to develop a comprehensive framework, we start by proposing five main components that need to make up the framework. These are, selection and identification, conceptual hybridization and development, verification and validation, experimentation and running, and expertise. The first four components are stemmed from the existing wisdom of developing simulation models. The issue of expertise does not necessarily feature with such importance in solo modeling, however, it does play a very important role in hybrid modeling. We find that most of the existing frameworks ignore this elements when selecting between different paradigms, yet it is evident that the choice if modeling is significantly influenced by the expertise of the modeler, i.e. dictating which paradigm to be the leading one.

\section{PHILOSOPHICAL FOUNDATIONS FOR HYBRID (BALABAN)}

Philosophy of science is concerned with what qualifies as science, its purpose, foundations, methods, and implications. Paradigms of science can be described by fundamental teleological, ontological, epistemological, and axiological believes. Fundamental believes associated with scientific communities can be often identified based on their purposes, taxonomies, definitions, theoretical basis, perspectives on objectivity and acceptable knowledge, and values. Fundamental system of definitions can build up a field of study as related to other field(s) or standing on its own based on a new origin. Tolk et al. (2013) discussed whether current philosophy of science is sufficient or a new pragmatic philosophy of simulation is needed. Modeling and Simulation (M\&S) is a multi-disciplinary arena with multiple, both qualitative and quantitative, methods used for various purposes. Because of this variety, pragmatism as philosophy of science seems the most appropriate (Balaban et al. 2014). Formulated by James (1975) pragmatic philosophy of science offers a mediating stance between positivism/post positivism and interpretivism/constructivism, which could be appropriate for M\&S in general, and especially vital for the type of research that involves multiple methods. As pointed out by Robey (1996) “...theories and methods are justified on pragmatic grounds as appropriate tools for accomplishing research aims"(406). Mingers 


\section{Eldabi, Balaban, Brailsford, Mustafee, Nance, Onggo, Sargent}

(2001) proposed to remove constraints related to paradigms at the level of methods directing the focus on methods, whether taken from an established method, often called a paradigm, or not. One can also observe that what pragmatic philosophy offers effectively aligns with what is advocated by Mingers (2001). One can draw a relation that a paradigm is or has one or more methods, while a method is not necessarily a paradigm. For a discussion about relation between paradigm and a method see (Balaban et al. 2014). Pragmatism permits choosing multiple views that promote achieving the best answer to research questions. Depending on the type of research question, observable and/or socially constructed meanings can add to a body of knowledge. Although pragmatism is more methodologically permissive, it should require additional verification of assumptions and tailored and more stringent evaluation when using multiple methods as compared to a single method type of research.

Teleological and ontological believes should define boundaries and principles on purpose of the field of study and what exists within the field, respectively. Balaban and Hester (2013) investigated reasoning behind the use of multiple methods based on both $M \& S$ literature and purposes of mixing methods from the empirical social science. Based on this investigation the concept of complementarity of methods stands out as it was pointed out by both social scientist Greene (2007) and M\&S oriented scholars Brailsford et al. (2003), Morecroft and Robinson (2005). Terms and definitions must set and support agreed upon research communication standards that aim to reduce ambiguity. Balaban et al. (2014) discussed evolution of the meaning of hybrid simulation beyond continuous and discrete methods. Based on reviewed literature it was observed that the term hybrid simulation is now more synonymous with the term multi-method, i.e. not limited to the two original methods. This can also be observed in the work of Powell and Mustafee (2014) who proposed to extend the term hybrid simulation into a hybrid M\&S study, offering a more holistic perspective on the use of multiple methods. In this extended scope, the meaning of the term hybrid M\&S study, if not fully synonymous, is very close in the meaning with the term multi-method M\&S approach advocated by Balaban et al. (2014). The ontological basis in relation to the approach with multiple methods were introduced by Balaban et al. (2014) proposing a system of relevant definitions, and were further expanded and refined by Balaban (2015b). Lynch and Diallo (2015) constructed a taxonomy to describe modeling terminologies with respect to the characteristics of their models and found that none of the modeling terminologies explicitly dealt with all categories of model characteristics.

Epistemological believes should guard a field by directing scholars on how knowledge can be attained, which also overlaps with axiological believes. Assuming knowledge as a central (intrinsic) value, axiological believes form an evaluative relation between the gained knowledge and the approach that was used to gain that knowledge. Both epistemology and axiology have consequences toward research methodology. Because of the multidisciplinary and multipurpose character of M\&S arena, methodological challenges may occur especially when multiple methods are considered within the same study boundaries. An approach that uses multiple $M \& S$ methods grandfathers all problems from within M\&S arena, with additional concerns related to abduction risks (Lorenz and Jost 2006). Several research guidelines for a hybrid M\&S study / multi-method M\&S approach were proposed (Lynch et al. 2014; Balaban 2015b). Epistemological concerns related to research guidelines can be more or less method and domain specific. This creates tradeoff situations, where more specific guidelines can be more robust and easier to implement but permit less flexibility and creativity in the modeling process. Following the pragmatic philosophical stance, Balaban et al. (2015) used multiple philosophical concepts including theory of falsification (Popper 2002) along with concepts of triangulation (Balaban 2015a), complementarity (Greene 2007; Balaban and Hester 2013), and commensurability (Balaban et al. 2015) to provide four general theoretical principles for justification of the use of multi-method M\&S approach. Following these theoretical principles Balaban (2015b) proposed research guidelines for multi-method conceptualization, which is both method and domain generic. Axiological considerations related to hybrid simulation or its extended versions ought to encompass evaluative aspects related to its value. Within a realm of philosophy of science, assuming monists' view and taking knowledge as an intrinsic value, a value of hybridization could be examined as intertwined with the purpose by assuming that the approach has a value because it was required to produce 
knowledge. In this case a merit of using multiple methods can be examined formally by showing inability to represent required system or phenomenon using a specific single method, which could be further supported by real world examples. Balaban (2015a) explored concept of triangulation as a way of scientific evaluation of $M \& S$ based research. He proposed multiple dimensions: 1) triangulation level as a starting point of a study, 2) investigators, 3) methods considered, 4) methods preselected, and 5) data. If triangulation could be used to investigate credibility of M\&S research, this arguably could provide an evaluative aspect of gained knowledge. If accumulated and analyzed, this can project and redefine a value of the M\&S research in general, and value of research in which multiple methods were used in particular.

This brief overview introduced philosophy of science as relevant for M\&S field in general, and for hybrid simulation community in particular. The future work should continue to clarify, refine and expand the ontological basis by investigating both taxonomy and system of definitions. Future work on epistemological basis should include refinements and expansion of principles for approach that uses multiple M\&S methods as needed. Improvements of these principles should lead to research guidelines minimizing biases, uncovering false assumptions, and promoting fairness and equity. In particular, the work is needed to define principles and a generic approach that would allow for verification of assumptions between methods, prevent abduction risks as discussed by Lorenz and Jost (2006). Future work should also investigate pluralistic view of axiological basis of hybridization, and how axiological basis could contribute to expansion of teleological basis.

\section{REPRESENTATION METHOD FOR A HYBRID SIMULATION CONCEPTUAL MODEL (ONGGO)}

A hybrid simulation model is a hierarchical model that comprises modules, the interface between modules and updating rules (Onggo 2014). Subsequently, a module is also a model. This definition highlights the hierarchical structure of a hybrid simulation model. Figure 1 shows two hybrid simulation examples (the plus sign indicates that the module or model element can be expanded). Model 1 represents a type of hybrid simulation model that is formed by simply connecting a number of modules. This model is paradigm-free, but each module may adopt a specific paradigm (e.g. module 2 is a DES model). Model 2 represents a hybrid simulation model that adopts a specific paradigm and at least one of its modules uses a different paradigm (e.g. model 2 is a SD model in which one of its flows is a DES model).

Conceptual modelling is an important step in simulation modelling, but the one that is least understood. There has been significant progress in the research on conceptual modelling for DES, as demonstrated by various sessions, panels and tutorials at the Winter Simulation Conference since 2010. A textbook on conceptual modelling has also been written (Robinson et al. 2010). Hybrid simulation has been gaining in popularity recently, and it has been a dedicated track at the Winter Simulation Conference since 2014. Hence, we have the opportunity to think about conceptual modelling for hybrid simulation early in its development (compare this to the significant time lag between the early days of DES and the critical mass of conceptual modelling research in DES). There has been some discussion about the conceptual modelling stage of a hybrid simulation study, e.g. Zulkepli and Eldabi (2015) look at the framework for hybrid simulation and Onggo (2014) looks at the representation of a hybrid simulation model. This work is still at an early stage.

Conceptual modelling is the process of abstracting a model from a real or proposed system into a conceptual model (Robinson 2010). At some point in a simulation project, a conceptual model needs to be communicated to relevant stakeholders. A good conceptual model representation should facilitate effective communication between stakeholders, as this is crucial for a successful simulation project. The importance of involving stakeholders is arguably higher in some applications (e.g. healthcare, see Brailsford 2005).

Representation Method: The main challenge in designing a method for conceptual model representation is to devise a representation that can be understood by all stakeholders and yet which remains expressive enough to handle the varying levels of complexity in the system. To complicate matters further, there is no single accepted definition of what a conceptual model is (Robinson 2010), as what is to be 
represented will surely affect its representation. To start the discussion, I will use Robinson's definition. He categorizes the components of a conceptual model into objectives, inputs, outputs, assumptions, simplifications and content (scope, level of detail and structure).
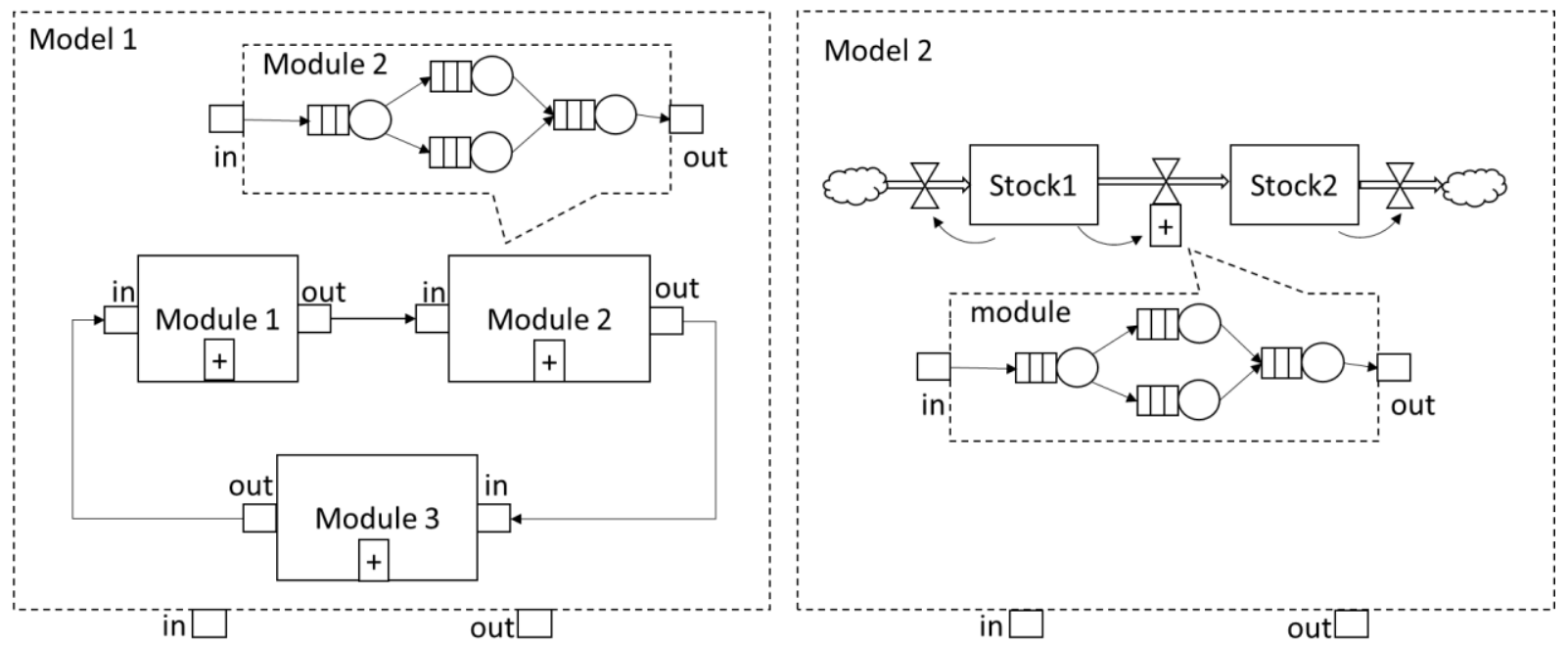

Figure 1: Two examples of hybrid simulation models

Objectives. This component documents the objective of a simulation study, which can be used to evaluate the success of the study and compare the quality of various decision alternatives. The representation of this component in a hybrid simulation is the same as in other simulation paradigms. Diagrams, such as an objective diagram (see Onggo 2009), can be used to represent this component.

Input and output. Once the objectives have been defined, we need to translate them into output variables that can be quantified. We also need to identify the different input variables that will be transformed into output variables by a hybrid simulation model that will be developed. By definition, a module that forms a hybrid simulation model is a simulation model in its own right. Hence, the input and output of a module can be represented as they are in a model (e.g. by using an influence diagram, see Onggo 2009). The difference is that, in hybrid simulation, we need to specify the interfaces between modules (see Model 1), or between a module and a model element (see Model 2). An interface defines the information that will be passed between modules, or between a module and a model element, which includes aggregation and disaggregation methods when applicable. Aggregation and disaggregation methods are needed when two modules (or a model element and a module) have different levels of detail (e.g. population and individual levels).

Assumptions and simplifications. Assumptions are used to address uncertainty or unknown factors that may be important in the model. Simplifications are used to handle the complexity of processes and other important elements (such as resources) in the model. Since each module in a hybrid simulation model is a model, tables or lists can be used to represent assumptions and simplifications (e.g. Robinson 2008).

Content (model boundary and level of detail). Figure 1 shows two examples of how the content of a hybrid simulation can be represented. A representation method for a hybrid simulation should support the hierarchical nature of the model, i.e. some elements or modules can be expanded or contracted (the plus signs in Figure 1). When a hybrid simulation model is formed by connecting a number of modules (e.g. Model 1 in Figure 1), a block diagram, as shown in Figure 1 (Model 1), is sufficient. When a hybrid simulation model adopts a specific paradigm (e.g. model 2 in Figure 1), an acceptable representation for the paradigm can be used but it should have a placeholder in which a module can be embedded in the model (e.g. the plus sign in Model 2 in Figure 1). Within each module, we can use the most common representation method for the chosen paradigm (e.g. a process-flow diagram for DES or a stock-and-flow diagram for 
SD). Unfortunately, ABS does not have a de facto representation method. A number of representation methods for ABS, such as flow charts, pseudocode, Petri Nets, DEVS, UML and BPMN, are discussed in Onggo (2013).

I have explained what a hybrid conceptual model should look like and how to represent them using existing methods. My main motivation is to start the discussion about conceptual modelling for hybrid simulation early in its development. Hence, we can design a standard (vendor-neutral) representation method that is expressive but easy to understand. It should also provide a seamless transition when we move from one paradigm to another as we look at the different modules in a hybrid simulation model. The main challenge is to find a well-tested representation method for a hybrid-simulation conceptual model. The method should be evaluated not only from the perspective of design principles but also from its usability (by real users).

\section{HYBRID SIMULATION IN A REAL-WORLD CONTEXT (BRAILSFORD)}

It is evident that the increasing popularity of hybrid simulation has highlighted the need for more formal approaches throughout the modeling process, starting with the conceptual modeling phase and moving through the implementation, verification and validation, experimentation and documentation phases. These phases or steps are relatively well understood for standalone discrete-event simulation (DES) models but become far more complex and challenging when DES is combined with other simulation methods. In this section I shall focus on model validation, and shall discuss some key challenges from the perspective of one specific application area, healthcare.

Hybrid simulation in health: Healthcare has been a particularly fruitful application area for hybrid simulation modeling. Many researchers have argued the need for combining DES and system dynamics (SD) in the context of healthcare systems, which are characterized by complexity, inter-connectedness and variability. As far back as 2003, Brailsford, Churilov and Liew (2003) argue that "ailing emergency departments" suffer from a variety of different problems which require treatment with a combination of DES and SD. Emergency departments (EDs) are stochastic queuing systems par excellence: it is impossible to avoid the impact of individual patient variation, and it is small wonder that so many DES models have been developed to try to improve patient flow and achieve the 4-hour target throughput time. However in reality, the real problems of managing an ED lie in the wider system outside the ED, both upstream (for example, how the ambulance service operates, and how performance metrics for ambulance crews may conflict with ED performance metrics) and downstream (the availability of beds, diagnostics and other resources in the hospital). A DES model might well "optimize" flow within the ED itself, but cannot properly take into account these other factors without growing into a mega-model of the whole hospital and the community it serves. Many of the hybrid models in the healthcare modeling literature fall into what Chahal and Eldabi (2008) term the Process-Environment category, recognizing that while a whole systems approach is essential for capturing the feedback dynamics in large, complex systems, the importance of individual patient variability cannot be ignored.

Validation in system dynamics: Validation of SD models has been recognized as a contentious area for decades. The founding father of system dynamics, Jay Forrester, as quoted in Sterman (2000), regarded SD models as "learning laboratories" for gaining understanding and insight, rather than numerical models to which standard statistical and other methods for comparing model output with observed data can be applied. Such methods can be applied to stock-flow models, where all the parameters are numerical and (arguably) objective, but the real strength of SD lies in its ability to capture qualitative variables which other modeling methods often ignore. However Forrester believed that at some level, the same could be said of all models. Chapter 21 of John Sterman's seminal textbook (2000) is entitled "Truth and Beauty: Validation and Model Testing" and contains the following quote (Forrester, 1961, p 123): "Any "objective" model-validation procedure rests eventually at some lower level on a judgment or faith that either the procedure or its goals are acceptable without objective proof". Section 21.1 of this chapter is entitled "Validation and Verification are Impossible". The real question we should be asking is, is this model 
useful? We know it is wrong (all models are wrong) but do its defects prevent it from being used to achieve some purpose, for someone? Thus validation of SD models typically involves many "softer" skills such as establishing face validity and involving stakeholders in all stages of the model development process, as well as the more technical but arguably less useful aspects such as checking dimensional consistency or performing extreme value tests. Validation of agent-based models: Agent-based models, especially those which model human behavior (as many healthcare applications do), face similar challenges. Such models often have a lot in common with SD models, in that they embody and/or encode the modeler's assumptions and beliefs about causality, the micro-level relationships between model elements and psychological behavioral rules, which often cannot ever be validated statistically at the individual level. In many agentbased models, the aim is essentially to invent sufficiently reasonable behavioral rules at the agent level such that when the model runs, the emergent behavior at population level replicates observed data. A good example of this is Billari et al.'s Wedding Ring model (2007) which sets out to model an individual's decision about when (and who) to marry, based on plausible assumptions about social pressure and other factors. The model output is then compared with actual administrative data on age at first marriage. The micro-level parameters in an ABM can be adjusted to achieve the best fit to observed data at macro level: this is a similar conceptual approach to the calibration of SD models, where a model is "tuned" so that it fits one or more known output variables. Philosophically, this is almost the diametrical opposite of validation, since it is manipulating the model to achieve the desired result.

Validation of hybrid models: If validation of standalone SD or ABS models is tricky enough, it all becomes infinitely more so when the SD or ABS model is combined as a hybrid with another kind of simulation. Viana (2014) and Viana et al (2014) discusses this issue in relation to a specific healthcare application, the sexually transmitted disease chlamydia. An epidemic model of the spread of the infection in a population is combined with a DES model of the hospital clinic in which patients get treated. In this case, the two models are totally separate: they were developed in different software packages and are linked by an Excel interface. The output from the SD (new cases of chlamydia) was transformed into the input for the DES (clinic arrivals). The output from the DES (untreated patients) was transformed into the input for the SD (general level of infection in the community), and the cycle repeats. The two models can be run independently, and were validated separately using standard methods for DES and indeed for SD, since this particular model is a stock-flow compartmental SIR (susceptible-infected-recovered) model for which the underpinning equations are well established in the literature. However, validating the hybrid (or "composite") model was a different matter, and in all honesty, was not fully addressed in either paper. It is intuitively clear that in reality, the DES and SD parts of this system are linked: poor clinic performance, i.e. a large number of (infected) patients leaving without being treated because waiting times are too long, will impact on the level of infection in the community and will ultimately generate more cases of chlamydia, who show up at the clinic and make the situation even worse. However, this entirely plausible assumption omits other links between the hospital and the community healthcare systems and is probably impossible to validate in practice. Viana et al (2014) compared the hybrid model to the standalone DES and concluded that it did give more credible results, in that it did at least acknowledge that the two healthcare systems are connected and affect each other.

Summary: I believe that validation of hybrid models in healthcare will remain a challenge for many years. The problem that "everything affects everything else" will always be true in healthcare, indeed increasingly so as health and social care become more integrated and organizations get more complex. Of course, the same is true for many other socio-economic systems, e.g. the criminal justice system. Given the increasing popularity of hybrid simulation, there is an urgent need for research in this area to address this challenge. My personal opinion, based on nearly 30 years' experience of model-building in healthcare applications, concurs $100 \%$ with Forrester's view: validation has more in common with marketing, or evangelism, than statistics. It is about identifying one's target audience and then persuading them to believe in your model ... by whatever means works best! 


\section{HYBRID SIMULATION: ROOTS AND RELATIONSHIPS (NANCE)}

When is the term "hybrid simulation" first used, appears in published form, and how is it defined? How does hybrid simulation relate to "combined simulation," strategic versus tactical simulation, analytic and closed-form modeling, or multi-model problem solving? Answers to such questions cannot be easily provided, and some would argue that neither the answers nor the questions are important.

The organization of this panel session is motivated by a desire to gain some historical perspective on the use of the term "hybrid simulation," the characterizations of the modeling approach, and the techniques viewed as constituting the methodology. The rationale is that lessons learned from a fuller, more complete, understanding of the roots might enable a clearer framing of the current challenges and a more accurate assessment of the future possibilities. This component seeks to identify and describe early research considered as hybrid simulation and to compare or contrast these efforts with others falling within the scope of problem-solving using modeling and simulation.

My awareness of the use of hybrid simulation to reduce execution time is triggered by the work of Schwetman (1978), in which a two-phase model execution uses steady-state approximations of job active times (using short-term resources) to set the multi-programming level that governs the simulation of longterm resources by arriving jobs. Comparative results of execution times with a simulation-only model show an impressive advantage for the hybrid simulation.

Prior interest in combined simulation, motivated by system evaluation objectives within accuracy requirements, are expanded by the recognition that solution efficiency and analytic and simulation interactions should be included. In a course entitled "Advanced Production Controls" in spring 1966, my opportunity to develop a system (nee industrial) dynamics model with a discrete event sub-model is realized. The assigned objective of comparing dispatching rules is expanded to an evaluation that extends beyond the production floor (the discrete event sub-model) to include order processing, warehousing, and customer sectors, with performance measures reflecting a system context.

A key paper distinguishes hybrid modeling from hybrid simulation and clarifies the relationships by defining four classes (Shantikumar and Sargent 1983). More specific commentary on this work is expected from a co-author and panel member. A decade later Sargent (1994) provides an informative history of hybrid simulation/analytic models and modeling, dividing his observations into three periods: (1) pre-1978, (2) 1978-84, and (3) post-1984. His observations conclude that little work in hybrid modeling is evident in the first period because of the lack of modeler sophistication. During the second period a flurry of activity occurs, but post-1984 activity is limited, with little recognition of the unifying classifications proposed in (Shantikumar and Sargent 1983). Finally, he attributes the lack of attention to hybrid simulation/analytic models and modeling to "primarily because this 'material' is not included in textbooks and is not taught in the classroom and secondarily it requires a certain amount of sophistication in modelers" (Sargent 1994, p. 386).

An alternative explanation of the absence of a rush to adoption of the unifying approach embodied in the four classes is that combined simulation, provoking considerably greater interest during 1974-94, is not explicitly treated. Also apparently ignored is the community that defines hybrid simulation as simulation model execution on a hybrid computer (Bratley, Fox and Schrage 1987, p. 24-25).

Sargent's lament at the lack of use of hybrid modeling and simulation in 1994 is certainly unwarranted some two decades later. A cursory search engine inquiry using "hybrid simulation," "hybrid model simulation," and minor variations with slightly different keywords leads to the following observations:

1. The predominant published work in the field is application focused among numerous widely ranging domains.

2. A scan of a sampling of documents from these domains indicates that the use of the terms "hybrid simulation" or "hybrid modeling" is not confined to solution approaches using analytic and simulation components.

3. The scan also indicates that the meaning and understanding of the terms "hybrid simulation" or "hybrid modeling" varies notably from one domain to another, and possibly within a single domain. 
4. Some papers apply the "hybrid" term to models that formerly would have been labeled "combined."

\section{A PERSPECTIVE ON HYBRID MODELS AND MODELING (SARGENT)}

This section gives the author's perspective on hybrid models and modeling. What is of interest here is when a model combines more than one option within a model subtype and/or uses more than one solution method; and thus is no longer a pure model with a pure solution method. The following discussions are going to be restricted to situations that have discrete-events as all or part of the model. Probability the first such model combination was a combined discrete-continuous model (simulation), which uses both differential equations and discrete events to describe a system that has both continuous phenomena and discrete events occurring in it. Tocher (Hollocks 2008) was probably the first individual to conduct the first combined discrete-continuous simulation that occurred in the mid-1960's, which was of a steel plant; although, Tocher apparently did not use the term combined model/simulation in his work (nor mixed discrete-continuous simulation). In 1970, Fahrland (1970) published perhaps the first article describing combined discretecontinuous models/simulations. Prikster was perhaps the person who make the term "combined discretecontinuous simulation" (also known as combined simulation) a commonly used term in the field of simulation through his book on GASP IV (Prikster 1974). It should be noted that in the United Kingdom (and perhaps elsewhere in Europe) the term used is mixed discrete-continuous models (Hollocks 2008, Pidd 2005) instead of combined discrete-continuous models; i.e., they use 'mixed' instead of 'combined' that is commonly used in the USA.

Perhaps the next significant use of more than one option in a model subtype occurred when more than one world view of DES models was introduced into the SLAM language (Prikster and Pegden 1979). DES models using more than one world view were not given any special label or called any special name. It should be noted that SLAM also allowed for continuous modeling as well as discrete modeling and thus one could have a combined discrete-continuous model using SLAM.

After developing models that combined DES models and analytic models (e.g., Shanthikumar and Sargent 1981), which are two options of structural models that are combined together, Shanthikumar and Sargent (1983) published an article that gave unifying definitions of both hybrid simulation/analytic models and modeling, where simulation is define to mean DES. The hybrid model definition is "A hybrid simulation/analytic model is a mathematical model which combines identifiable simulation and analytic models" and the hybrid modeling definition is "Hybrid simulation/analytic modeling consists of building independent simulation and analytic models of the total system, developing their solution procedures, and using their solution procedures together for problem solving." One major difference between hybrid simulation/analytic models and modeling is that hybrid modeling requires the simulation and analytic models to be independent whereas for hybrid models they only need to be identifiable; and a second difference is the specific solution procedure required of hybrid modeling. Hybrid models have their simulation and analytic models and their solution procedures combined in some way. Both hybrid models and modeling involve simulation and analytic models and how their solutions work.

Four classes of hybrid models are given by Shanthikumar and Sargent (1983). The specifics of the classes will not be discussed here except for Class II hybrid models. In Class II hybrid models, the solution procedure has the simulation and analytic models moving through time together and interacting with each other over time as appropriate. Thus, most combined discrete-continuous simulations would belong to this class of hybrid models. Similarly, if a DES and a SD model (recall that SD is an analytic model) had a solution procedure moving them through time together and interacting with each other as appropriate, then this hybrid model would be a Class II hybrid model. Since continuous models and SD models are analytic models, they can be used in hybrid simulation/analytic models and in simulation/analytic modeling. The four classes of hybrid models can aid in developing hybrid models. (Shanthikumar and Sargent (1983) also contain four usages of hybrid simulation/analytic modeling that can aid in hybrid modeling.)

Hybrid simulation/analytic models and modeling have been used since the beginning of DES. Tocher's combined discrete-continuous simulation discussed above is a Class II hybrid model. Shanthikumar and 
Sargent (1983) give references to others who have used these types of model and modeling and give new examples. There were panel sessions (Sargent et al. 1982 and 1984) at the 1982 and 1984 Winter Simulation Conferences that discussed hybrid simulation/analytic models and modeling.

In 1994, Sargent (1994) gave a historical view of hybrid simulation/analytic models and modeling primarily based on reviewing the literature to find the amount of activity that occurred regarding hybrid models and modeling. Based on his investigation, Sargent divided time into three periods based on the amount of activity that was occurring: pre-1978, 1978-1984, and post-1984. In the pre-1978, there were only a few examples found in the literature of hybrid models and hybrid modeling and these were by sophisticated modelers. During the time period of 1978-1984, there was a considerable amount of activity in (a) the applications of hybrid simulation/analytic models and modeling and (b) promoting and developing systematic approaches to hybrid models and modeling. In the post-1984 period, there were only a limited number of applications of hybrid models and modeling found in the literature. Updating Sargent's 1994 paper, we find that we should end the third time period and start a fourth period. Thus we will define the third time period to be1985-2011 and the fourth time period being post-2011. Beginning in 2012 and thereafter we find that the amount of activity regarding hybrid models and modeling increasing year-toyear in both (i) applications and (ii) new methods and systematic approaches. A WSC track for hybrid models began in 2014 and continues to date. Much of the recent interest in hybrid models is (a) using DES/SD hybrid models, (b) occurring within Europe, and (c) happening within the health care field. It will be interesting to see if this new interest in hybrid models continues into the future or if dies out as it did in the 1980's.

To summarize, this author believes that the unifying definitions of hybrid simulation/analytic models and modeling and the four classes of hybrid models developed by Shanthikumar and Sargent (1983) are still applicable. This author also believes the use of a set of standard definitions for hybrid models and modeling would be useful for communication, understanding, and developing hybrid models and modeling and suggests that the definitions of Shanthikumar and Sargent (1983) and their four classes of hybrid models be put into current practice.

\section{Hybrid Systems Modelling Approach: Extending M\&S Methodology to Inform Practice (MUSTAFEE)}

The application of multiple techniques in the model implementation/coding stage of a simulation study is referred to as Hybrid Simulation, which is distinguished in this position piece from Hybrid Systems Modelling Approach, the latter referring to studies that apply inter-disciplinary methods and techniques from disciplines like Economics and Applied Computing (Powell and Mustafee, 2014). The position being offered by the panellist is, Hybrid Systems Modelling Approach (subsequently referred to as Hybrid Approach or only HA) extends M\&S methodology (including hybrid simulation) by combining approaches from across disciplines, thereby adding further value to the conventional M\&S study and its application to practice. Based on the discipline-specific methods and what it has to offer, this added value gained could be mapped to various stages of a simulation study. For example, Mustafee and Bischoff (2013) have used HA for gaining added value in the model experimentation stage through the combined application of load plan construction heuristics with agent-based simulation; Powell and Mustafee (2014) have discussed the use of qualitative systems dynamics in the conceptual modelling phase; Mustafee and Taylor (2009) and Mustafee et al. (2009) have used HA for faster execution of experiments using distributed computing approaches like desktop grid computing and distributed simulation respectively. Thus, while the focus of the panel is on Hybrid Simulation, it is argued that the next stage of development of M\&S will come from the increasing use of inter-disciplinary methods (Mustafee et al., 2015). A defining factor of HA is its reliance on inter-disciplinary research in the methodology space; this is not usually the case for Hybrid Simulation. An example of HA using game theory is provided next. The methodological extension to a traditional M\&S study is discussed followed by an example of blood supply chain. Readers may like to refer to Katsaliaki, Mustafee and Kumar (2014) which provides an overview of the blood supply chain and 
the use of serious games (note however that the game being referred to in this paper is different to games that are the subject of this paper).

Game-theoretic approaches in model development, validation and simulation experimentation: M\&S study starts with a real-world problem/consideration for a future system, a conceptual model is then developed and validated, followed by the implementation of a computer model. In the verification stage the computer model is checked to ensure that it is a good representation of the conceptual model and is implemented correctly. Experiment scenarios are then developed and verified; the scenarios are experimented. Finally, and subsequent to the process of ensuring operational validation, the results of the simulation may be implemented. Using similar constructs it is possible to relate to the stages of a game theoretic study. Like a conventional M\&S study, game theoretic modelling may start with a real world problem and the identification of players in the system. A theoretical model is developed followed by a reduced form model (mathematical model); this is then implemented as a game. A game theorist relies on lab and field experiments wherein the players play these games (usually an incentive is provided to the players). This captures user behavior and provides understanding of how the players are likely to behave in a real world situation.

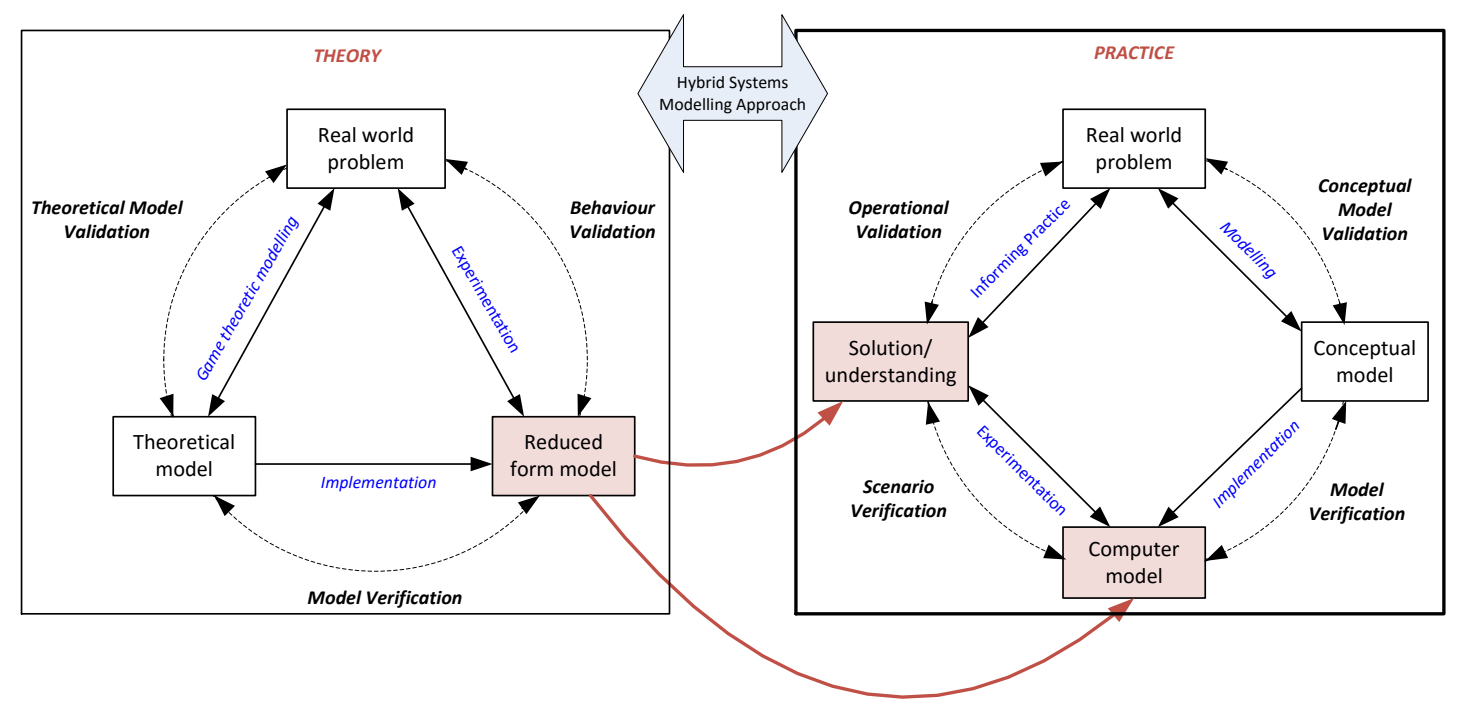

Figure 3: Hybrid Systems Modelling Approach using Game Theory

What do we gain from their combined application? Whereas M\&S study presents us with well-defined stages for conceptual and operational validation, in the game theoretic paradigm we gain from behavior validation. A HA using game theory and M\&S will enable development of models which may better represent the actors in a service system. Taking an example from healthcare and the blood supply chain, the actors may be those responsible for policy at NHS Blood and Transplant (NHS BT), blood manufacturing units, blood centers which stock and issue blood, hospitals which order blood on behalf of the doctors. A model focusing on blood ordering strategies to reduce wastage whilst ensuring stock availability, e.g., Katsaliaki and Brailsford (2007), will often model the actors without due consideration to their behavior in real-life; using the game theoretic terminology we may say that the model is developed based on the assumption that the players are cooperating. This may not be the case in reality. For example, hospitals may try to game the system by ordering more blood than is necessary. It is arguable that a model developed using non-realistic assumptions will have fewer chances of successful implementation. A better outcome may be achieved by gaining an understanding of user behavior (captured using theoretical models) and then developing a computer simulation model and experimental sub-scenarios based not only on the assumption of cooperative players (as is the case traditionally), but also players that are non-cooperating. For each experiment scenario this would allow a range of sub-scenarios to be compared and contrasted; this 
will further aid decision making. Taking the example of two players in the system (hospital 1 and hospital 2), the best case scenario can be conceptualized as, (a) both hospitals being honest about their requirement for blood units, (b) hospital 1 is over-claiming, (c) hospital 2 is over-claiming, and (d) both hospitals are over-claiming. As shown in the Figure 3, a HA approach as discussed here will extend M\&S methodology and will inform practice. Considering the plethora of non-M\&S techniques that are used in Operations Research (Mustafee and Katsaliaki, 2016), Computer Science/Applied Computing, Psychology, Data Science, and so on so forth, HA is a fertile area of enquiry!

Acknowledgement: Nav would like to acknowledge the contribution of Dr. Surajeet Chakravarty (Department of Economics, Exeter University) towards the development of the methodology.

\section{REFERENCES}

Balaban, M. A. 2015a. "Credibility of Modeling and Simulation Via Triangulation." In MODSIM World Conference and Expo, edited by

Balaban, M. A. 2015b. "Toward a Theory of Multi-Method Modeling and Simulation Approach." Ph.D., Old Dominion University, Ann Arbor.

Balaban, M. A., and P. Hester. 2013. "Exploration of Purpose for Multi-Method Simulation in the Context of Social Phenomena Representation." In Winter Simulation Conference, edited by R. Pasupathy, S.H. Kim, A. Tolk, R. Hill, and M. E. Kuhl, 1661-1672. Piscataway, New Jersey: IEEE.

Balaban, M. A., P. Hester, and S. Y. Diallo. 2014. "Towards a Theory of Multi-Method M\&S Approach: Part I." In Winter Simulation Conference, edited by A. Tolk, S. Y. Diallo, I. O. Ryzhov, L. Yilmaz, S. Buckley, and J. A. Miller, 1652-1663. Piscataway, New Jersey: IEEE.

Balaban, M. A., P. Hester, and S. Y. Diallo. 2015. "Towards a Theory of Multi-Method M\&S Approach: Part Iii." In Winter Simulation Conference, edited by L. Yilmaz, H. K. Chan, I. C. Moon, T. Roeder, C. M. Macal, and M. D. Rossetti, Piscataway, New Jersey: IEEE.

Billari F.C., A. Prskawetz, Aparicio Diaz B., and Fent T. (2007). The "Wedding-Ring": An agent-based marriage model based on social interaction. Demographic Research 17(3):59-82

Brailsford, S., L. Churilov, and S. K. Liew. 2003. "Treating Ailing Emergency Departments with Simulation: An Integrated Perspective." In Health Sciences Simulation, edited by J. Anderson, and E. Katz. San Diego, USA: Society for Modeling and Computer Simulation.

Brailsford S (2005). Overcoming the barriers to implementation of operations research simulation models in healthcare. Clinical and Investigative Medicine 28:6, 312-315.

Brooks, R.J. and Robinson, S. (2000). Simulation. Palgrave Macmillan.

Chahal K and Eldabi T (2008). Applicability of hybrid simulation to different modes of governance in UK healthcare. Proceedings of the 2008 Winter Simulation Conference, Miami, Florida. S. J. Mason, R. R. Hill, L. Mönch, O. Rose, T. Jefferson, and J. W. Fowler, eds.

Fakhimi, M., Mustafee, N. and Stergioulas, L. (2015). "An Investigation of Hybrid Simulation for Modeling Sustainability in Healthcare." 2015 Winter Simulation Conference, December 6 - 9, 2015, Huntington Beach, CA. pp. 1585-1596.

Forrester, J. 1961. Industrial Dynamics. MIT Press, Cambridge. Mass.

Fahrland, D. A. 1970. "Combined Discrete Event Continuous Systems Simulation." SIMULATION 14 (2):61-72.

Greene, J. C. 2007. Mixed Methods in Social Inquiry. San Francisco, CA: Jossey-Bass

Helal, M., L., Rabelo, J. Sepúlveda, and A. Jones. 2007. "A Methodology for Integrating and Synchronizing the System Dynamics and Discrete Event Simulation Paradigms." In Proceedings of the 25th International Conference of the System Dynamics Society, edited by J. D. Sterman, M. P. Repenning, R. S. Langer, J. I. Rowe, and J. M. Yarni.3:1-24. Boston, MA System Dynamic Society. 
Hollocks, B. W. 2008. "Intelligence, Innovation, and Integrity—K. D. Tocher and the Dawn of Simulation." Journal of Simulation 2: 128-137.

James, W. 1975. Pragmatism. Vol. 1: Harvard University Press.

Katsaliaki, K., \& Brailsford, S. C. (2007). Using simulation to improve the blood supply chain. Journal of the Operational Research Society, 58(2), 219-227.

Katsaliaki, K., Mustafee, N. and Kumar, S. (2014). A Game-based Approach towards Facilitating Decision Making for Perishable Products: An Example of Blood Supply Chain. Expert Systems with Applications, 41(9): 4043-4059.

Lorenz, T., and A. Jost. 2006. "Towards an Orientation Framework in Multi-Paradigm Modeling." In Conference of the System Dynamics Society, edited by A. Globler, E. A. J. A. Rouwette, R. S. Langer , J. I. Rowe, and J. M. Yanni, 23-27.

Lynch, C., J. Padilla, S. Diallo, J. Sokolowski, and C. Banks. 2014. A Multi-Paradigm Modeling Framework for Modeling and Simulating Problem Situations. Proceedings of the 2014 Winter Simulation Conference.

Lynch, C. J., and S. Y. Diallo. 2015. A Taxonomy for Classifying Terminologies That Describe Simulations with Multiple Models. Proceedings of the 2015 Winter Simulation Conference.

Lynch, Christopher J., and Saikou Y. Diallo. "A taxonomy for classifying terminologies that describe simulations with multiple models." In Proceedings of the 2015 Winter Simulation Conference, pp. 1621-1632. IEEE Press.

Mingers, J. 2001. "Combining Is Research Methods: Towards a Pluralist Methodology." Information Systems Research 12 (3):240.

Morecroft, J., and S. Robinson. 2005. "Explaining Puzzling Dynamics: Comparing the Use of System Dynamics and Discrete-Event Simulation." In 23rd International Conference of the System Dynamics Society, edited by 17-21.

Morgan, J., Howick, S. and Belton, V. 2011. Designs for the complementary use of system dynamics and discrete-event simulation. In Proceedings of the Winter Simulation Conference. Edited by S. Jain, R.R. Creasey, J. Himmelspach, K.P. White, and M. Fu. 2715-2727. Piscataway, New Jersey: IEEE Winter Simulation Conference.

Mustafee, N. and Taylor, S.J.E. (2009). "Speeding Up Simulation Applications Using WinGrid". Concurrency and Computation: Practice and Experience, 21(11): 1504-1523.

Mustafee, N. and Bischoff, E.E. (2013). "Analysing Trade-offs in Container Loading: Combining Load Plan Construction Heuristics with Agent-based Simulation". International Transactions in Operational Research, 20(4): 471-491.

Mustafee. N. and Katsaliaki, K. (2016). "A Keyword Classification Scheme for OR/MS and M\&S." In Proceedings of the 2016 Spring Simulation Multi-Conference (SpringSim'14) - ANSS symposia, April 3 - 6, 2016, Pasadena, CA. Society for Modelling and Simulation International (SCS).

Mustafee, N., Brailsford, S.C., Diallo, S., Padilla, J., Powell, J.H., and Tolk, A. (2015). "Hybrid Simulation Studies and Hybrid Simulation Systems: Definitions, Challenges, and Benefits." 2015 Winter Simulation Conference, December 6 - 9, 2015, Huntington Beach, CA. pp. 1678-1692.

Mustafee, N., Taylor, S.J.E., Katsaliaki, K. and Brailsford, S. (2009). "Facilitating the Analysis of a UK National Blood Service Supply Chain Using Distributed Simulation". SIMULATION: Transactions of the Society of Modelling and Simulation International, 85(2): 113-128.

Onggo BSS (2009). Towards a unified conceptual model representation: A case study in healthcare. Journal of Simulation 3:40-49.

Onggo BSS (2013). Agent-Based Simulation Model Representation Using BPMN, in Fonseca, P. et al. (Eds), Formal Languages for Computer Simulation: Transdisciplinary Models and Applications. Hershey, PA: Information Science Reference, pp. 378-399

Onggo BSS (2014). Elements of a hybrid simulation model: A case study of the blood supply chain in lowand middle-income countries. Proceedings of the 2014 Winter Simulation Conference, 1597-1607. 
Pidd, M. 2005. Computer Simulation in Management Science, $5^{\text {th }}$ ed. New York: John Wiley and Sons. Popper, K. 2002. The Logic of Scientific Discovery. London and New York: Routledge

Powell, J., and N. Mustafee. 2014. Soft or Approaches in Problem Formulation Stage of a Hybrid M\&S Study. Proceedings of the 2014 Winter Simulation Conference.

Prikster, A. A. B. 1974. The GASP IV Simulation Language. New York: John Wiley and Sons, Inc.

Prikster, A. A. B. and C. D. Pegden. 1979. Introduction to Simulation and SLAM. New York: John Wiley and Sons, Inc.

Robey, D. 1996. "Research Commentary: Diversity in Information Systems Research: Threat, Promise, and Responsibility." Information systems research 7 (4):400-408.

Robinson S, Brooks R, Kotiadis K. and van der Zee D-J. (2010) Conceptual Modelling for Discrete-Event Simulation. Boca Raton, FL: Taylor and Francis.

Robinson S (2010) 'Conceptual modelling for simulation: Definition and requirements'. In: Robinson S., Brooks R., Kotiadis K. and van der Zee, D-J. (Eds), Conceptual Modelling for Discrete-Event Simulation. Boca Raton, FL: Taylor and Francis, pp. 235-256.

Robinson S (2008). Conceptual Modelling for Simulation Part 1: Definition and Requirements. Journal of the Operational Research Society 59(3): 278-290.

Sargent, R. G. 1994. "A Historical View of Hybrid Simulation/Analytic Models," In Proceedings of the 1994 Winter Simulation Conference, edited by J. D. Tew, M. S. Manivannan, D. A. Sadowski, and A. F. Seila, 383-386. Piscataway, New Jersey: Institute of Electrical and Electronics Engineers, Inc.

Sargent, R. G. 2015. "Types of Models." Chapter 5 in Modeling and Simulation in the Systems Engineering Life Cycle, edited by M. L. Loper, 51-55. London: Springer-Verlag.

Sargent, R.G. (2005). "Verification and validation of simulation models." Proceedings of the 37th conference on Winter simulation. winter simulation conference, 2005.

Sargent, R. G., M. Rosenhine, S. Duket, J. G. Shankthikumar, and C. H. White. 1984. "What Type of Model: Analytic, Simulation, or Hybrid Simulation/Analytic," Only title and authors listed under Panels in Proceedings of the 1984 Winter Simulation Conference, edited by S. Sheppard, U. Pooch, and D. Pedgen. Piscataway, New Jersey: Institute of Electrical and Electronics Engineers, Inc.

Sargent, R. G., G. T. Sparow, J. G. Shanthikumar, and W. R. Lilegdon. 1982. "Panel on Hybrid Simulation/Analytic Models and Modelling," In Proceedings of the 1982 Winter Simulation Conference, edited by H. J. Highland, Y. W. Chao, and O. S. Madrigal, 696. Piscataway, New Jersey: Institute of Electrical and Electronics Engineers, Inc.

Shanthikumar, J. G. and R. G. Sargent. 1981. A Hybrid Simulation/Analytic Model of a Computerized Manufacturing System," In Operational Research 81 (Proceedings of the Ninth International IFORS Conference on Operational Research) edited by J. P. Brans, 901-915. Amsterdam: North Holland.

Shanthikumar, J. G. and R. G. Sargent. 1983. "A Unifying View of Hybrid Simulation/Analytic Models and Modeling," Operations Research 31 (6): 1030-1052.

Sargent R G (2013), An introduction to verification and validation of simulation models. Proceedings of the 2013 Winter Simulation Conference, R. Pasupathy, S.-H. Kim, A. Tolk, R. Hill, and M. E. Kuhl, 321-327. Piscataway, New Jersey: IEEE.

Sterman, J. D. 2000. Business dynamics: systems thinking and modeling for a complex world. Irwin/McGraw-Hill, Boston.

Tolk, A., B. Heath, M. Ihrig, J. Padilla, E. Page, E. Suarez, C. Szabo, P. Weirich, and L. Yilmaz. 2013. "Epistemology of Modeling and Simulation." In Winter Simulation Conference, edited by R. Pasupathy, S.-H. Kim, A. Tolk, R. Hill, and M. E. Kuhl, 1152-1166. Piscataway, New Jersey: IEEE.

Viana J, Brailsford S C, Harindra V and Harper P R 2014. Combining discrete-event simulation and system dynamics in a healthcare setting: A composite model for Chlamydia infection, European Journal of Operational Research 237: 196-206. 
Viana J 2014. Reflections on two approaches to hybrid simulation in healthcare. Proceedings of the 2014 Winter Simulation Conference, Savannah, GA. A. Tolk, S. Y. Diallo, I. O. Ryzhov, L. Yilmaz, S. Buckley, and J. A. Miller, eds.

Zulkepli, J. and Eldabi, T. 2015. Towards a framework for conceptual model hybridization in healthcare. In Winter Simulation Conference, edited by L. Yilmaz, H. K. Chan, I. C. Moon, T. Roeder, C. M. Macal, and M. D. Rossetti, Piscataway, New Jersey: IEEE

\section{AUTHOR BIOGRAPHIES}

TILLAL ELDABI is a Senior Lecturer at Brunel University. His email address is tillal.eldabi@brunel.ac.uk.

MARIUSZ A. BALABAN is an M\&S Scientist/Engineer at MYMIC. His email addresses are mariusz.balaban@mymic.net and mariusz.balaban@outlook.com.

SALLY C. BRAILSFORD is Professor of Management Science at the University of Southampton, UK. Her email address is s.c.brailsford@soton.ac.uk.

NAVONIL MUSTAFEE is Senior Lecturer at University of Exeter Business School. His e-mail address is n.mustafee@exeter.ac.uk his webpage is http://sites.google.com/site/navonilmustafee/.

RICHARD E. NANCE is an Emeritus Professor of Computer Science at Virginia Tech. His e-mail address is nance@vt.edu.

BHAKTI STEPHAN ONGGO is Lecturer in the Department of Management Science at the Lancaster University Management School, UK. His email address is s.onggo@lancaster.ac.uk.

ROBERT G. SARGENT is a Professor Emeritus of Syracuse University. His e-mail is rsargent @syr.edu. 